\title{
Non-invasive monitoring of fiber fermentation in healthy volunteers by analyzing breath volatile metabolites: lessons from the FiberTAG intervention study
}

\author{
Audrey Neyrinck \\ UCLouvain \\ Julie Rodriguez \\ UCLouvain \\ Zhengxiao Zhang \\ University of Alberta
}

Benjamin Seethaler

University of hohenheim

Florence Mailleux

UCLouvain

Joeri Vercammen

Interscience

Laure B. Bindels

UCLouvain

Patrice D. Cani

UCLouvain

Julie-Anne Nazare

Université de Lyon

Véronique Maquet

Kitozyme

Martine Laville

Université de Lyon

Stephan C. Bischoff

University of Hohenheim

Jens Walter

University of Alberta

Nathalie Delzenne ( $\square$ nathalie.delzenne@uclouvain.be)

Universite catholique de Louvain Ecole de Biologie https://orcid.org/0000-0003-2115-6082 
Research

Keywords: gut microbiota, chitin-glucan, fermentation, breath volatile metabolites

Posted Date: August 24th, 2020

DOI: https://doi.org/10.21203/rs.3.rs-62395/v1

License: (c) (i) This work is licensed under a Creative Commons Attribution 4.0 International License. Read Full License 


\section{Abstract}

Background: The fermentation of dietary fibers (DF) leads to the production of bioactive metabolites, the most volatile ones being excreted in the breath. The aim of this study was to analyze the profile of exhaled breath volatile metabolites (BVM) and gastro-intestinal symptoms in healthy volunteers after a single ingestion of maltodextrin (placebo) versus chitin-glucan (CG), an insoluble DF previously shown to be fermented into short-chain fatty acids (SCFA) by the human microbiota in vitro.

Methods: $4.5 \mathrm{~g}$ maltodextrin (day 0) or $4.5 \mathrm{~g} \mathrm{CG}$ (day 2) were added to a standardized breakfast in fasting healthy volunteers $(n=15)$. BVM were measured using selected ion flow tube mass spectrometry (SIFTMS) throughout the day, as well as gastrointestinal tolerance by using validated visual analogue scale. Faecal gut microbiota (Illumina 16S rRNA sequencing) and SCFA (gas chromatography with flameionization detection) were analyzed prior to intervention.

Results: A single ingestion of $4.5 \mathrm{~g} \mathrm{CG}$ did not induce significant gastrointestinal discomfort. Untargeted metabolomics analysis of breath highlighted that 13 MS-fragments (among 408 obtained from ionizations of breath) discriminate $C G$ versus maltodextrin acute intake in the postprandial state. Targeted analysis revealed that CG increased exhaled butyrate, but also 5 other BVM - including the microbial metabolites 2,3-butanedione and 3-hydroxybutanone - with a peak observed $6 \mathrm{~h}$ after CG intake. Correlation analyses spotlighted Mitsuokella as a potential genus responsible for the presence of butyric acid, triethylamine and 3-hydroxybutanone in the breath.

Conclusion: Measuring BMV in the breath reveals the microbial signature of the fermentation of DF after a single ingestion. This standardized protocol allows to analyze the time-course of released bioactive metabolites that could be proposed as new biomarkers of DF fermentation, potentially linked to their biological properties.

\section{Background}

Dietary fibers (DF) are non-digestible polysaccharides considered as beneficial for human health, namely through their effect on gut function such as the modulation of stool production (1). They are classified according to their chemical structure, their food source, their water solubility and viscosity, or their fermentability (1). The categorization of DF as viscous soluble (e.g. pectin), non-viscous soluble (e.g. inulin) or as insoluble (e.g. cellulose) is an attempt to link their physico-chemical properties to physiological effects. However, this may link to erroneous conclusions regarding their fermentability by the gut microbiota. Indeed, several insoluble DF can be fermented by gut microbes, and could thus be considered as potential prebiotics $(1,2)$. A prebiotic refers to a substrate that is selectively utilized by host microorganisms conferring a health benefit (3). Chitin-glucan (CG) is an insoluble DF considered as safe food ingredient by the European Food Safety Authority (4). It is extracted from the cell walls of the mycelium of Aspergillu nigerfungi and is composed of branched $\beta-1,3 / 1,6$ glucan linked to chitin via a $\beta-1,4$ linkage. Marzorati et al. demonstrated that CG was fermented with enhanced short-chain fatty acids 
(SCFA) production (mainly propionate and butyrate) using an in vitro approach (Simulator of the Human Intestinal Microbial Ecosystem) (5). In previous studies in rodents, we and others showed that CG significantly improved metabolic parameters- an effect linked to an increase of butyrate-producing Roseburia spp. and reduced cardiovascular risk factors $(6,7)$. In humans, CG administration for 6 weeks reduced oxidized low-density lipoproteins $(\mathrm{LDL})$ in the serum, the mechanism behind this observation being unexplored (8).

The identification of bioactive metabolites produced upon fermentation of DF in the gut is important to afford the biological events involved in health improvement, and to propose biomarkers of nutrient intake. Volatile organic compounds (VOCs) in the breath have been proposed as potential surrogate markers of gut dysbiosis in gastrointestinal diseases and diabetes mellitus $(9,10)$. Bacteria being a major source of volatile metabolites, it was suggested that breath volatile metabolites (BVM) such as $\mathrm{H}_{2}$, SCFA or alkanes may be an indicator of host health $(11,12)$.

In the context of the project FiberTAG (Joint Programming Initiative "A Healthy Diet for a Healthy Life" 2017-2020 https://www.fibertag.eu/), we aimed to identify bioactive bacterial metabolites linking DF intake and gut microbiota-related health effect (13). In this present study, exhaled BVM have been analyzed by selective ion flow tube mass spectrometry (SIFT-MS, $(10,14)$ ) in healthy volunteers to monitor the potential interaction of a single exposure to $C G$ as insoluble DF with the gut microbiota.

\section{Materials And Methods Participants}

The FiberTAG study is an interventional monocentric study including two test days aiming at characterizing the fermentation of CG (Kiotransine ${ }^{\circledR}$ from KitoZyme, Belgium) versus maltodextrin (Cargill, Belgium), a non-fermentable digestible carbohydrate given as placebo, by assessing the volatile compounds released in the breath. For this purpose, blinded subjects received a single administration of maltodextrin $(4.5 \mathrm{~g})$ and two days later, a single administration of CG $(4.5 \mathrm{~g})$. After the single administration of CG fiber or maltodextrin given together with the breakfast, the kinetic of BVM production was measured repeatably during twelve hours. Healthy subjects were recruited by the Center of Investigation in Clinical Nutrition (CICN-UCLouvain) by displaying posters on the university site and by mails, by social networks, local newspapers and local flyers disposed in shops and doctor's offices in nearby cities. Subjects were pre-screened by phone or mail using a questionnaire to test if they met the inclusion criteria listed in Additional file 1. The participants were invited to perform a screening test using lactulose to select $\mathrm{H}_{2}$-producers. Forty subjects passed the screening lactulose test at least 4 weeks before the intervention. Briefly, fasted subjects (at least 10 hours) received on oral load of $10 \mathrm{~g}$ of lactulose; then, exhaled $\mathrm{H}_{2}$ was measured every 30 minutes for 4 hours using Lactotest 202 (Medical Electronic Construction, MEC). A minimal increase of $10 \mathrm{ppm}$ of $\mathrm{H}_{2}$ during 3 successive measurements was considered as the criteria of subject selection. No increase should be observed in the first 30 minutes to avoid subjects with small intestinal bacteria overgrowth. After the screening test, 15 subjects were 
selected respecting inclusion and exclusion criteria (Additional file 1) and were subjected to medical examination with the physician investigator to verify that they were healthy. At least 12 days before the first test day, the volunteers were asked by a dietician to follow dietary recommendations to avoid eating fiber-rich food products containing high amounts of prebiotic DF more than once a week. These foods comprised, among others whole grains, artichokes, Jerusalem artichokes, salsifies, leeks, onions.

Prebiotics and probiotics supplementation as well as the consumption of fiber-enriched-food were forbidden. The subjects were asked to complete a food diary for 3 days the week before the intervention. The Nubel Pro program and the table of composition from Nubel 2010 were used to assess macronutrient and total fiber intake. Up to 4 days before the intervention period, stool samples were collected and stored directly at $-20^{\circ} \mathrm{C}$ for further transport within 4 days to the $\mathrm{CICN}$ for storage at $-80{ }^{\circ} \mathrm{C}$ until analyses. This study was approved by the local ethical committee (Comité d'Ethique HospitaloFacultaire UCLouvain/Cliniques Universitaires Saint-Luc) and written informed consent was obtained from all subjects. The trial was carried out in accordance with the Good Clinical Practice as required by the following regulations: the Belgian law of 7 May 2004 regarding experiments on the human persons and the EU Directive 2001/20/EC on Clinical Trials (registration at clinicaltrials.gov as NCT03494491).

\section{Intervention days}

Both test days were organized in the same way. An overview of the study design is shown in Fig. 1. Subjects were blinded regarding the compound they received. The evening before the two intervention days, subjects were asked to eat rice and meat, without vegetables and to avoid alcohol. Fasted subjects (from $9 \mathrm{pm}$ the day before) arrived at $7 \mathrm{am}$ at the $\mathrm{CICN}$. The study coordinator reviewed the inclusion and exclusion criteria and verified if instructions were adequately followed. After mouth wash (with Perio Aid Intensive Care), breath samples were taken to analyze BVM at baseline. In the meantime, subjects were asked to complete a visual analogue scale (VAS) questionnaire about the 8 gastrointestinal symptoms usually described after the consumption of fibers. Then subject received a standardized breakfast composed of white bread and butter, with $240 \mathrm{ml}$ of water and $4.5 \mathrm{~g}$ of CG or the placebo. The breakfast should be eaten within $15 \mathrm{~min}$. Breath samples were collected after $30 \mathrm{~min}$ and then every 2 hours during 12 hours after the CG or placebo ingestion to measure BVM. In the meantime, every hour, subjects were asked to complete the VAS questionnaire. Of note, 2 hours after the CG or placebo ingestion, subjects were free to drink water. Five hours after the start, the subjects received a standardized lunch composed of white bread, ham and cheese. At 4 pm (8 hours after the start) subjects received a collation (sweet waffle). The test day ended at $8 \mathrm{pm}$.

\section{Gastrointestinal symptoms}

The 100-mm VAS about the eight gastrointestinal (cramps, bloating, rumbling, discomfort, flatulence, burps, gastrointestinal reflux and nausea) was filled out at baseline and every hour for 12 hours after the ingestion of CG or maltodextrin load. The scales were scored by measuring the distance (in $\mathrm{mm}$ ) from 0 with a ruler. Scores were expressed as changes from baseline in $\mathrm{cm}$.

\section{Breath volatile metabolites (BVM) analysis}


Breath samples were collected into bags before and 30 minutes after the ingestion of CG or the placebo and then every 2 hours for 12 hours. The bags were directly sent to Interscience (Louvain-La-Neuve, Belgium) for BVM measurement by gas chromatography using a TCD detector (for $\mathrm{H}_{2}$ analysis every $4 \mathrm{~h}$ ) and using selected ion flow tube mass spectrometry (SIFT-MS for other BVM). For this last methodology, the reagent ions $(\mathrm{H} 3 \mathrm{O}+, \mathrm{NO}+$ and $\mathrm{O} 2+)$ were generated by a microwave air discharge at $0.5 \mathrm{Torr}$, selected by using a quadrupole mass filter and injected into a stream of carrier gas in the flow tube. All the ion products of the chemical ionization reaction and un-reacted reagent ions were monitored by a quadrupole mass spectrometer in the full scan mode in the mass-to-charge ratio $(\mathrm{m} / \mathrm{z})$ range of $10-200$ over $60 \mathrm{~s}$. Bags filled with helium were used as blank. The full scan data (ion counts per second) averaged over the sampling time for each $\mathrm{m} / \mathrm{z}$ value was used for the statistical analyses LabSyft software package (version 1.4.4, Syft Technologies) was used for the mass spectrum acquisition and data exportation as comma-separated values (CSV) files. In total, three data sets were obtained from $\mathrm{H} 3 \mathrm{O}+, \mathrm{NO}+$ and $\mathrm{O} 2+$ ionizations. We targeted volatile metabolites known to study energy metabolism and to study the gutrelated metabolic effects of DF, in particular those known to be a reflect of gut fermentation (coming from bacterial metabolism) $(11,12)$. Identified BVM were expressed as changes from baseline in ppm.

\section{Gut microbiota analysis}

Bacterial DNA was extracted from faecal samples from baseline (before interventions) using the QIAamp DNA Stool Mini Kit (QIAGEN, Hilden, Germany), as previously described (15). All samples of this study were sequenced in the same run. Alpha diversity indexes and beta diversity indexes were calculated using QIIME2. PCA plot of the beta-diversity indexes were visualized using $\mathrm{R}$ software. The raw sequencing data is deposited into the Sequence Read Archive (SRA) of NCBI (http://www.ncbi.nlm.nih.gov/sra) under BioProject PRJNA636138.

\section{Faecal SCFA analysis}

SCFA were analysed in the native faecal samples as previously described (15).

\section{Data integration and statistical analysis}

Data are expressed as means \pm SEM. Baseline values were compared by matched-pairs Wilcoxon signedrank test; to adjust for multiple testing, the $p$-value of the Wilcoxon test was adjusted with a false discovery rate (FDR) of $5 \%$ according to the Benjamini-Hochberg procedure (q-value, significant if $q<$ 0.05). Net area under the curve (AUC) were calculated for each symptom recorded every hour and for each identified BVM recorded every two hours after maltodextrin or CG intake. Wilcoxon matched-pairs signed rank test were used to compare net AUC. In addition, repeated measures ANOVA were performed to compare evolution of BVM over time. For the gut microbiota analysis, relative abundances performed in Qiime2 are expressed as means and SEM and were calculated on $\mathrm{R}$ for each taxon. To avoid analyzing spurious sequences, bacteria taxa with an average relative abundance below $0.1 \%$ in all samples were removed. PCA and PLS-DA models were built based on selected variables in R (v3.5.1, package "ade4" and "mixOmics"). For PLS-DA, a loading $>0.9$ was chosen. For PCA, statistical differences among group were determined by Monte Carlo permutations to handle non-parametric data distributions $(n=999$, 
implemented in R). Correlation analysis was performed between BVM and all genera identified, by Spearman's correlation test using the "corrplot" package in R. A significance level of $p<0.05$ (q-value for adjusted p-value, using FDR correction ) was adopted for all analyses.

\section{Results}

\section{Characterization of the subjects (baseline faecal gut microbiota, SCFA composition and hydrogen production)}

The interventions were conducted in March 2018. Fifteen subjects were selected as $\mathrm{H}_{2}$-producers based on the lactulose test (Additional file 2) and completed the study. The mean age of participants (seven men and eight women) was 21 years and their mean body mass index was $22.1 \pm 0.5 \mathrm{~kg} / \mathrm{m}^{2}$. Before the intervention, the mean energy intake was $2046 \pm 130 \mathrm{kcal} /$ day and their nutrient intakes were $229 \pm 23$, $81 \pm 6,70 \pm 5$ and $20 \pm 4 \mathrm{~g}$ per day for carbohydrates, lipids, proteins and fiber, respectively.

The overall composition of gut microbiota was assessed by the measure of both a-diversity and $\beta$ diversity indexes. Subjects seemed to display a different gut microbiota composition as shown by the principal coordinate analysis of the Bray-curtis index; subject I being the most distant from others (Fig. 2a). However, this difference was reduced when the distance measure considers the fraction of branch length in a phylogenic tree, as assessed by the unweighted uniFrac distance; subject $\mathrm{G}$ being the most distant from others on this PCoA. Alpha-diversity related to bacterial richness (Observed OTU), evenness (Pielou) or both (Shannon) was systematically lower for subject I (Fig. 2b). Gut microbiota analysis presented at the phylum level confirmed the lower a-diversity found for this subject for which Verrucomicrobia and Tenericutes were absent from their bacterial profile in faeces (Fig. 2c). Those phyla were also absent in the faecal matter of subject $\mathrm{N}$ who presented also low a-diversity indexes. Firmicutes was the most abundant bacterial phylum for all subjects $(>60 \%)$ followed by either Bacteroidetes or Actinobacteria depending on the subject. The total amount of SCFA in the faecal matter are presented in Fig. 2d. Subjects $E$ and $G$ exhibited the highest levels of faecal SCFA whereas subject $L$ presented the lowest total amount of faecal SCFA. We noted lower proportions of valeric and iso-valeric acids in subject I as compared to other subjects. Those SCFA were not detectable for subject C (Fig. $2 \mathrm{~d}$ ). The total amount of SCFA in all subjects varied between $1 \mathrm{mg} / \mathrm{g}$ to $4 \mathrm{mg} / \mathrm{g}$ of faecal dry matter, acetate being the most abundant one.

\section{Gastrointestinal tolerance after CG versus maltodextrin ingestion in healthy subjects}

In order to evaluate the tolerance of ingesting CG after a unique intake during the breakfast, VAS scores were recorded. The baseline scores of gastrointestinal parameters between the 2 test days were not significantly different (Additional file 3). The evolution of the different scores throughout the day was not 
significantly affected by GC intake, suggesting a good gastrointestinal tolerance (Fig. 3). Only flatulence was more important as soon as $6 \mathrm{~h}$ after CG intake, as compared to maltodextrin intake ( $p=0.054)$.

\section{BVM profile after single ingestion of CG or maltodextrin in healthy subjects}

The SIFT-MS analysis was carried out using three precursor ions $(\mathrm{H} 3 \mathrm{O}+, \mathrm{NO}+$ and $\mathrm{O} 2+)$ to record the resulting fingerprints of the breath (untargeted analysis) (Fig. 4a). PCA analysis of MS-fragments in overnight fasted state (time $0 \mathrm{~h}$ ), in pre-prandial state meaning before lunch (time $4 \mathrm{~h}$ ) and in postprandial-post absorptive state (time $6 \mathrm{~h}$ ) showed that the 15 subjects formed separate clusters depending on the time of measurement, and therefore on the nutritional status (fasted, pre-prandial, post-prandial). The clustering at time $6 \mathrm{~h}$ was more pronounced in CG than in the maltodextrin group, indicating a difference in BVM profile between both treatments (Fig. 4b). Before the lunch (time $4 \mathrm{~h}$ ), the trends were distinct from the overnight fasting state (time $0 \mathrm{~h}$ ) and we observed a slight overlap between both groups (Fig. 4b). Interestingly, after the lunch at time $6 \mathrm{~h}$, a clear separation (from PLSDA analysis) appeared for participants that received CG compared to subjects receiving maltodextrin and was linked to 13 MSfragments (Fig. 4c). Targeted analysis was conducted to identify 26 BVM known to be involved in energy metabolism in particular those coming from bacterial metabolism $(11,12)$. The concentrations (in ppm) of all BVM exhaled in the breath over time for both test days are presented in Additional file 4.

Consistently with the untargeted analysis, we observed that only one BVM exhaled in the breath (pentane) was more important at the test day with maltodextrin (Additional file 5). All other BMV had similar value in fasted state upon both days (day 0 and day 2). Repeated measures ANOVA revealed a significant effect for time and for subject inter-variation for all targeted BVM $(p<0.05)$. A significant effect of the treatment was obtained only with butyric acid, triethylamine, 2,3-butanedione, 3-hydroxybutanone, ethane, methanol and pentane (repeated measures ANOVA $p<0.05$ ). Figure 5 presents the evolution of exhaled SCFA over time corrected by the baseline value at fasted state. Among all SCFA identified, the evolution of propionic acid and butyric acid in the breath was significantly affected after a single ingestion of CG. Of note, the level of exhaled propionic acid was lower after CG intake than maltodextrin intake whatever the time considered. More importantly, exhaled butyric acid increased after CG intake after the lunch and reached the highest level at time $6 \mathrm{~h}$ in post-prandial state compared to participants ingesting maltodextrin. The level of exhaled butyrate (at time $6 \mathrm{~h}$ and the net AUC) after CG intake was not correlated with the initial concentration of faecal butyrate ( $r=0.40$ and $r=-0.07$, respectively; $p>0.05)$. Baseline Mitsuokella relative abundance was the sole genus positively correlated with net AUC of exhaled butyrate (Additional file 4). The evolution of other exhaled SCFA were not significantly different between the 2 test days. Figure 6 revealed other BVM with an AUC that was significantly affected after CG versus maltodextrin intake: triethylamine, 2,3-butanedione, 3-hydroxybutanone $(p=0.054)$ and pentane followed the same trends than the butyric acid with a peak observed at time $6 \mathrm{~h}$ for triethylamine, 3hydroxybutanone and 2,3-butanedione, and $8 \mathrm{~h}$ for pentane. Of note, net AUC of triethylamine was positively correlated with Mitsuokella and Catenibacterium; 3-hydroxybutanone was positively correlated to Mitsuokella, Alloprevotella, Lachnospiraceae FCSO20 group and Ruminococcaceae NK4A214 group 
whereas exhaled 2,3-butanedione was positively correlated with Muribaculaceae metagenome, Clostridium sensu stricto and Flavonifractor (Additional file 4). Methanol increased whereas ethane decreased just after CG-breakfast intake and a peak was observed at $8 \mathrm{~h}$ for methanol. Although some of them were increased after $\mathrm{CG}$ ingestion, the evolution of well-known markers of gut fermentation such as exhaled $\mathrm{H}_{2}$ or $\mathrm{CH}_{4}$ as well as other targeted BVM were not significantly affected after $\mathrm{CG}$ compared to maltodextrin intake (Additional file 6). We observed that the highest levels of acetone, acetonitrile, acetic acid, propionic acid, caproic acid, 1- and 2-propanol, benzene and benzaldehyde were found at time 0 (meaning in the fasting state) since all changes from baseline were negative after treatment and throughout the test day for those metabolites (Fig. 5, Additional file 6). In accordance with the untargeted analysis, several identified BVM such as acetic acid, (iso)valeric acids, acetone, acetonitrile, ethanol, butanol, isoprene, methane and $\mathrm{H}_{2} \mathrm{~S}$ were different between the pre- and postprandial states (time $4 \mathrm{~h}$ versus time $6 \mathrm{~h}$ ) without any significant effect of CG treatment. Isoprene was the sole BVM which decreased after lunch in both groups (Fig. 5, Additional file 6).

\section{Discussion}

Effects of DF are usually investigated using urine, blood and faecal analysis, but exhaled breath is of increasing interest because it allows non-invasive sampling. There are almost 900 VOCs in the exhaled breath of healthy humans (16) which could be considered as potential biomarkers of metabolic disorders, specific diets, or both. Consistently, breath analysis (mainly hydrogen and methane) is already used in clinics for monitoring gastrointestinal disorders (12,17-19). Recently, Rondanelli et al. reviewed biomarkers present in exhaled breath of gastrointestinal diseases and nutritional status (20). For example, exhaled acetone has been reported to be an important biomarker of diabetes (9). A few studies have also monitored the effects of diet (gluten-free diet, high versus low fat dairy drink, high versus low fiber diets) on the composition of exhaled breath $(12,21-24)$. In the present study, we compared for the first time the effect of a unique ingestion of an insoluble DF (CG prone to be fermented by the gut microbiota) versus maltodextrin (a fully digestible carbohydrate) as placebo on BVM profile in young healthy adults. The interpretation of our data considered characterization prior intervention in terms of faecal microbiota composition and faecal SCFA. We hypothesized that some BVM may be the reflect of DF gut fermentation with respect to inter-subject variation.

It has already been described that the presence of some metabolites such as acetone, ethanol, isoprene and methanol in the breath is conditioned by the fasting state (11). In our study, the highest levels of acetone but also acetonitrile, acetic acid, propionic acid, caproic acid, 1- and 2-propanol, benzene and benzaldehyde were found at fasting state in the morning. The potential origin of such BVM was already discussed elsewhere focusing on diet, environmental exposure (exposure to air pollutants) and biological pathways $(11,25)$. Some of them were decreased after the breakfast without any further variation due to the lunch, the collation or the CG intake whatever the time considered (1- and 2-propanol, benzene and benzaldehyde). We hypothesized that the presence in breath at time $0 \mathrm{~h}$ of this kind of BVM with high affinity with fat tissue may be the result of the high rate of lipolysis at fasted state. Other BVM were 
different between the pre- and postprandial states without any significant influence of CG intake. It is the case of acetic acid, (iso)valeric acids, acetone, acetonitrile, ethanol, isoprene, methane and $\mathrm{H}_{2} \mathrm{~S}$ for which levels increased after the lunch in both groups. Acetone, produced via fatty acid oxidation, has been one of the earliest identified gaseous disease biomarkers, being distinctively present in the breath of patients with diabetic ketoacidosis, hypoinsulinemic states, and starvation (26). Subtle acetone fluctuations also systematically occur in response to modest, physiological changes in insulin/glucose levels (26). In contrast, ethanol is not produced by human cells, but small concentrations in blood and breath derive from alcoholic fermentation of glucose by gut bacteria expressing pyruvate decarboxylase. Following carbohydrate ingestion, blood and breath ethanol increase (26). Furthermore, the levels of exhaled breath ethanol increased after intravenously administered glucose load, supporting the hypothesis that glucose enrichment of gastrointestinal capillaries per se may stimulate gut bacterial fermentation to some degree (27). The source and physiological effect of isoprene in humans are matters of debate. In animals and humans, this intermediary metabolite of cholesterol synthesis is formed in the liver from isopentenyl pyrophosphate (IPP) and its isomer (DMAPP) (28). However, as this reaction is slow and may be insignificant at physiological $\mathrm{pH}$ values, it is unlikely to completely explain the endogenous isoprene production by animals. So far, two major metabolic pathways leading to DMAPP have been identified: the mevalonic acid (MVA) pathway and the 1-deoxy-D-xylulose-4-phosphate/2-C-methylerythriol 5-phosphate (DOXP/MEP) pathway. The DOXP/MEP pathway was demonstrated to prevail in plants and most bacteria, whereas the MVA pathway is mainly present in higher eukaryotes. In bacteria DMAPP is converted into isoprene enzymatically by isoprene synthase. Surprisingly, isoprene increased with the time of fasting and fell down in postprandial state independently of the DF intake. Another study showed that isoprene concentrations did not change significantly following feeding with a liquid protein-energy meal (29). It would be interesting to assess the role of isoprene as marker of cholesterol synthesis, that can be affected differently following meal composition. A pilot study of 7 healthy subjects suggested that increased metabolic activity in the gastrointestinal tract after ingestion of a meal could explain, at least partly, the increase of BVM such as ethanol, 1-propanol, 3-hydroxybutanone, propionic acid, and butyric acid (12). Here, we did not observed changes with meal of propionic acid and 1-propanol. Anyway, our data led us to conclude that a lot of BVM from our targeted analysis, principally produced by host metabolism, reflected metabolic state rather than a response to a DF ingestion. In addition, our study revealed that bacterial metabolites coming from the gut microbiota and exhaled in the breath such as $\mathrm{H}_{2} \mathrm{~S}$, methane and SCFA (acetic acid, butyric acid, (iso)valeric acids, propionic acid, caproic acid) may be influenced by the ingestion of a meal or by the fasting state.

Hexose and pentose sugars are fermented by isolated human colonic bacteria via pathways leading to the formation of SCFA, ethanol and hydrogen depending on the strain and species (30). In particular, butyrate formation occurs in Firmicutes bacteria, either via butyrate kinase (in many Clostridium and Coprococcus species) or via butyryl CoA:acetate CoA transferase (31). Marzorati et al have shown that fermentation of CG led to an increased production of both propionate and butyrate in vitro (5). In the present study, a single intake of $4.5 \mathrm{~g}$ CG led to an increase of exhaled butyrate suggesting that this SCFA is not restricted to enterocyte metabolism, but may be absorbed in the circulation and then released in 
breath as a signature of gut fermentation of CG, since the increase did not appear when volunteers received placebo. We were unable to relate exhaled butyrate to faecal butyrate. It should be kept in mind that SCFA production mainly occurs in the proximal part of the colon where the availability of substrates is most abundant. Accordingly, up to $95 \%$ SCFA are rapidly absorbed by the colonocytes resulting in decreasing concentrations from the proximal to distal colon and only about $5 \%$ is excreted in faeces (32). In addition, intestinal microbial fermentation is a dynamic process influenced by a wide range of factors. Therefore, the levels of each metabolite are a result of metabolic fluxes of highly variable rates, which are not adequately represented in steady-state metabolite profiles in faeces. Our kinetic analysis offered the possibility to assess the dynamic processes of gut fermentation. Interestingly, exhaled butyrate was positively correlated with Mitsuokella from the Firmicutes phylum. It has been demonstrated that concentration of exhaled methanol increased from a physiological level of $\sim 0.4 \mathrm{ppm}$ up to $\sim 2 \mathrm{ppm}$ a few hours after eating around $500 \mathrm{~g}$ of fruits, the effect being principally due to pectin fermentation $(11,33)$. Here, the basal levels of breath methanol were in the same range than the physiological level but did not reach a concentration higher than $0.5 \mathrm{ppm}$. In addition, bacterial fermentation in the mouth or throat can partly explain increased postprandial levels of exhaled breath BVM (12). Breath methanol increased already just after taking the $C G$ with the breakfast, suggesting that this metabolite was not a reflect of CG fermentation in the lower part of the gut. The kinetics of triethylamine release was different, since it increased $4 \mathrm{~h}$ after CG intake with a peak obtained at time $6 \mathrm{~h}$ as observed for butyric acid but the range of changes was very weak (maximum $0.005 \mathrm{ppm}$ ). Although no literature exists about metabolic pathway explaining a potential origin from gut bacteria, the kinetic study performed in the present study supported the hypothesis that this BVM appeared following gut fermentation of CG as observed for butyric acid. We found that production in the breath was positively correlated with the presence of Mitsuokella and Catenibacterium in the faecal matter. Breath ethane decreased after CG intake whereas pentane followed the same kinetics than exhaled butyric acid. The presence of pentane in exhaled breath is considered as a result of lipid peroxidation of polyunsaturated fatty acids in cellular membranes, a process mediated by free radicals and oxidative stress (10). In contrast to butyrate, those considerations do not allow to state that the increases of pentane and methanol due to CG ingestion come mostly from direct bacterial metabolism. Data show that lactobacilli can produce 3-hydroxybutanone (acetoin) and 2,3-butanedione (diacetyl) (34). The production of 3-hydroxybutanone and 2,3-butanedione has already been studied on different Lactobacillus strains growing on Jerusalem artichoke juice rich in inulin that served as substrate (34). Their production is related to citrate metabolism through the citrate-oxaloacetate-pyruvateacetolactate-acetoin/diacetyl pathway, where pyruvate is considered as the precursor. In our study, although 3-hydroxybutanone and 2,3-butanedione were exhaled after CG ingestion, no correlation was found with lactobacilli. Interestingly, 3-hydroxybutanone production in the breath was positively correlated with Mitsuokella, the same bacteria revealed for butyric acid production. The genus Mitsuokella, comprising only two named species, are Gram-negative obligate anaerobes which utilize fermentable carbohydrates to form acetate, lactate and succinate (35). Of these two species, $M$. multacida is regularly found in the intestinal tract of humans and appears to be responsive to diet changes. The acids produced were potentially available to other acid-utilising bacteria for the formation of butyrate, such as Roseburia (36).

Page $11 / 22$ 
In conclusion, this study showed that CG intake changed the exhaled BVM profile and that meal intake influenced the BVM profile. Even if the limitation of the study is to focus on a small cohort and refer to hydrogen producers only, we have validated a protocol allowing to analyse the effect of DF intake in the morning on the profiling of breath metabolites throughout the day, showing that post prandial - post absorptive period is probably the most relevant timing for the evaluation of BVM. Exhaled butyric acid, triethylamine, 3-hydroxybutanone and 2,3-butanedione are reflecting the bacterial metabolism of CG. This implies that analysis of BVM in exhaled air can be used as a dynamic approach to noninvasively assess the responses to DF intake related to gut fermentation.

\section{Declarations}

\section{Acknowledgments}

We are very grateful to Barbara Pachikian from the UCLouvain platform CICN (Center of Investigation in Clinical Nutrition) for her helpful support and criticism during the preparation of the study and the investigation with volunteers. We thank Remi Selleslagh and Véronique Allaeys for the excellent technical assistance. We also thank the volunteers who participated in this study.

\section{Author's contributions}

Conceptualization: NMD, AMN, JAN, VM, ML, SCB, JW

Data curation: AMN, JR, NMD

Formal analysis: AMN, JR, ZZ, BS, FM, JV

Funding acquisition: NMD, PDC, VM, ML, SCB, JW

Investigation: AMN, JR, FM, NMD

Methodology: AMN, JR, ZZ, BS, FM, JV, LBB, JAN, ML, SCB, JW

Project administration: AMN, JR, NMD

Resources: PDC, NMD, SCB, JW

Software: ZZ, AMN, JR

Supervision: NMD

Validation: NMD, AMN, JR, LBB, ZZ

Visualization: JR, AMN 
Writing - original draft: AMN, JR, NMD

Writing - review \& editing: AMN, JR, NMD, ZZ, BS, PDC, LBB, JAN, VM, ML, SCB, JW

All authors read and approved the final manuscript.

\section{Funding}

FiberTAG project was initiated from a European Joint Programming Initiative "A Healthy Diet for a Healthy Life" (JPI HDHL). This work was supported by the Service Public de Wallonie (SPW-EER, convention 1610365, Belgium). NMD is a recipient of a grant from Belgium National Scientific Research Fund (FRSFNRS, convention PINT-MULTI R.8013.19 (NEURON, call 2019) and convention PDR T.0068.19) and from UCLouvain (Action de Recherche Concertée ARC18-23/092). PDC is supported by the Fonds Baillet Latour (Grant for Medical Research 2015), the Fonds de la Recherche Scientifique (FNRS, FRFS-WELBIO: WELBIO-CR-2019C-02R, and EOS program no.30770923).

\section{Availability of data and materials}

The raw sequencing data is deposited into the Sequence Read Archive (SRA) of NCBI (http://www.ncbi.nlm.nih.gov/sra) under BioProject PRJNA636138.

\section{Ethics approval and consent to participate}

See ethics paragraph in the "Materials and methods" section

\section{Consent for publication}

Not applicable

\section{Competing interests}

The other authors declare that they have no competing interests.

\section{References}


1. Stephen AM, Champ MM, Cloran SJ, Fleith M, van Lieshout L, Mejborn H, et al. Dietary fibre in Europe: current state of knowledge on definitions, sources, recommendations, intakes and relationships to health. Nutr Res Rev. 2017;30(2):149-90.

2. EFSA. Scientific opinion on the substantiation of health claims related to dietary fibre (ID 744,745 , $746,748,749,753,803,810,855,1415,1416,4308,4330)$ pursuant to Article 13(1) of Regulation (EC)No 1924/20061. EFSA Journal. 2010;8: 1735.

3. Gibson GR, Hutkins R, Sanders ME, Prescott SL, Reimer RA, Salminen SJ, et al. Expert consensus document: The International Scientific Association for Probiotics and Prebiotics (ISAPP) consensus statement on the definition and scope of prebiotics. Nat Rev Gastroenterol Hepatol. 2017;14(8):491502.

4. EFSA Panel on Dietetic Products NaAN. Scientific Opinion on the safety of 'Chitin-glucan' as a Novel Food ingredient. EFSA Journal. 2010;8:1687.

5. Marzorati MM, V; Possemiers,S. Fate of chitin-glucan in the human gastrointestinal tract as studied in a dynamic gut simulator (SHIME). Journal of Functional Foods. 2017;30:313-20.

6. Neyrinck AM, Possemiers S, Verstraete W, De Backer F, Cani PD, Delzenne NM. Dietary modulation of clostridial cluster XIVa gut bacteria (Roseburia spp.) by chitin-glucan fiber improves host metabolic alterations induced by high-fat diet in mice. J Nutr Biochem. 2012;23(1):51-9.

7. Berecochea-Lopez A, Decorde K, Ventura E, Godard M, Bornet A, Teissedre PL, et al. Fungal chitinglucan from Aspergillus niger efficiently reduces aortic fatty streak accumulation in the high-fat fed hamster, an animal model of nutritionally induced atherosclerosis. J Agric Food Chem. 2009;57(3):1093-8.

8. Bays HE, Evans JL, Maki KC, Evans M, Maquet V, Cooper R, et al. Chitin-glucan fiber effects on oxidized low-density lipoprotein: a randomized controlled trial. Eur J Clin Nutr. 2013;67(1):2-7.

9. Das S, Pal S, Mitra M. Significance of Exhaled Breath Test in Clinical Diagnosis: A Special Focus on the Detection of Diabetes Mellitus. J Med Biol Eng. 2016;36(5):605-24.

10. Rieder F, Kurada S, Grove D, Cikach F, Lopez R, Patel N, et al. A Distinct Colon-Derived Breath Metabolome is Associated with Inflammatory Bowel Disease, but not its Complications. Clin Transl Gastroenterol. 2016;7(11):e201.

11. Ajibola OA, Smith D, Spanel P, Ferns GA. Effects of dietary nutrients on volatile breath metabolites. J Nutr Sci. 2013;2:e34.

12. Raninen KJ, Lappi JE, Mukkala ML, Tuomainen TP, Mykkanen HM, Poutanen KS, et al. Fiber content of diet affects exhaled breath volatiles in fasting and postprandial state in a pilot crossover study. Nutr Res. 2016;36(6):612-9.

13. Neyrinck AM, Rodriguez J, Vinoy S, Maquet V, Walter J, Bischoff SC, et al. The FiberTAG project: Tagging dietary fibre intake by measuring biomarkers related to the gut microbiota and their interest for health. Nutrition Bulletin. 2020;45:59-65.

14. Boshier PR, Marczin N, Hanna GB. Repeatability of the measurement of exhaled volatile metabolites using selected ion flow tube mass spectrometry. J Am Soc Mass Spectrom. 2010;21(6):1070-4. 
15. Rodriguez J, Neyrinck AM, Zhang Z, Seethaler B, Nazare JA, Robles Sánchez C, et al. Metabolite profiling reveals the interaction of chitin-glucan with the gut microbiota. Gut Microbes 2020 (in press).

16. de Lacy Costello B, Amann A, Al-Kateb H, Flynn C, Filipiak W, Khalid T, et al. A review of the volatiles from the healthy human body. J Breath Res. 2014;8(1):014001.

17. Rezaie A, Buresi M, Lembo A, Lin H, McCallum R, Rao S, et al. Hydrogen and Methane-Based Breath Testing in Gastrointestinal Disorders: The North American Consensus. Am J Gastroenterol. 2017;112(5):775-84.

18. Smolinska A, Tedjo DI, Blanchet L, Bodelier A, Pierik MJ, Masclee AAM, et al. Volatile metabolites in breath strongly correlate with gut microbiome in CD patients. Anal Chim Acta. 2018;1025:1-11.

19. Raman M, Ahmed I, Gillevet PM, Probert CS, Ratcliffe NM, Smith S, et al. Fecal microbiome and volatile organic compound metabolome in obese humans with nonalcoholic fatty liver disease. Clin Gastroenterol Hepatol. 2013;11(7):868-75 e1-3.

20. Rondanelli M, Perdoni F, Infantino V, Faliva MA, Peroni G, lannello G, et al. Volatile Organic Compounds as Biomarkers of Gastrointestinal Diseases and Nutritional Status. J Anal Methods Chem. 2019;2019:7247802.

21. Kistler M, Szymczak W, Fedrigo M, Fiamoncini J, Hollriegl V, Hoeschen C, et al. Effects of diet-matrix on volatile organic compounds in breath in diet-induced obese mice. J Breath Res. 2014;8(1):016004.

22. Baranska A, Tigchelaar E, Smolinska A, Dallinga JW, Moonen EJ, Dekens JA, et al. Profile of volatile organic compounds in exhaled breath changes as a result of gluten-free diet. $J$ Breath Res. 2013;7(3):037104.

23. Barros R, Moreira A, Fonseca J, Delgado L, Castel-Branco MG, Haahtela T, et al. Dietary intake of alpha-linolenic acid and low ratio of n-6:n-3 PUFA are associated with decreased exhaled NO and improved asthma control. Br J Nutr. 2011;106(3):441-50.

24. Hageman JHJ, Nieuwenhuizen AG, van Ruth SM, Hageman JA, Keijer J. Application of Volatile Organic Compound Analysis in a Nutritional Intervention Study: Differential Responses during Five Hours Following Consumption of a High- and a Low-Fat Dairy Drink. Mol Nutr Food Res. 2019;63(20):e1900189.

25. Filipiak W, Ruzsanyi V, Mochalski P, Filipiak A, Bajtarevic A, Ager C, et al. Dependence of exhaled breath composition on exogenous factors, smoking habits and exposure to air pollutants. $J$ Breath Res. 2012;6(3):036008.

26. Lee J, Ngo J, Blake D, Meinardi S, Pontello AM, Newcomb R, et al. Improved predictive models for plasma glucose estimation from multi-linear regression analysis of exhaled volatile organic compounds. J Appl Physiol (1985). 2009;107(1):155-60.

27. Bolyen E, Rideout JR, Dillon MR, Bokulich NA, Abnet CC, Al-Ghalith GA, et al. Reproducible, interactive, scalable and extensible microbiome data science using QIIME 2. Nat Biotechnol. 2019;37(8):852-7.

28. King J, Mochalski P, Unterkofler K, Teschl G, Klieber M, Stein M, et al. Breath isoprene: muscle dystrophy patients support the concept of a pool of isoprene in the periphery of the human body. 
Biochem Biophys Res Commun. 2012;423(3):526-30.

29. Smith D, Spanel P, Davies S. Trace gases in breath of healthy volunteers when fasting and after a protein-calorie meal: a preliminary study. J Appl Physiol (1985). 1999;87(5):1584-8.

30. Russell WR, Hoyles L, Flint HJ, Dumas ME. Colonic bacterial metabolites and human health. Curr Opin Microbiol. 2013;16(3):246-54.

31. Louis P, Flint HJ. Diversity, metabolism and microbial ecology of butyrate-producing bacteria from the human large intestine. FEMS Microbiol Lett. 2009;294(1):1-8.

32. Verbeke KA, Boobis AR, Chiodini A, Edwards CA, Franck A, Kleerebezem M, et al. Towards microbial fermentation metabolites as markers for health benefits of prebiotics. Nutr Res Rev. 2015;28(1):4266.

33. Taucher J, Lagg A, Hansel A, Vogel W, Lindinger W. Methanol in human breath. Alcohol Clin Exp Res. 1995;19(5):1147-50.

34. Zalan Z, Hudacek J, Toth-Markus M, Husova E, Solichova K, Hegyi F, et al. Sensorically and antimicrobially active metabolite production of Lactobacillus strains on Jerusalem artichoke juice. J Sci Food Agric. 2011;91(4):672-9.

35. Gorham JB, Kang S, Williams BA, Grant LJ, McSweeney CS, Gidley MJ, et al. Addition of arabinoxylan and mixed linkage glucans in porcine diets affects the large intestinal bacterial populations. Eur J Nutr. 2017;56(6):2193-206.

36. Duncan SH, Hold GL, Barcenilla A, Stewart CS, Flint HJ. Roseburia intestinalis sp. nov., a novel saccharolytic, butyrate-producing bacterium from human faeces. Int J Syst Evol Microbiol. 2002;52(Pt 5):1615-20.

\section{Figures}




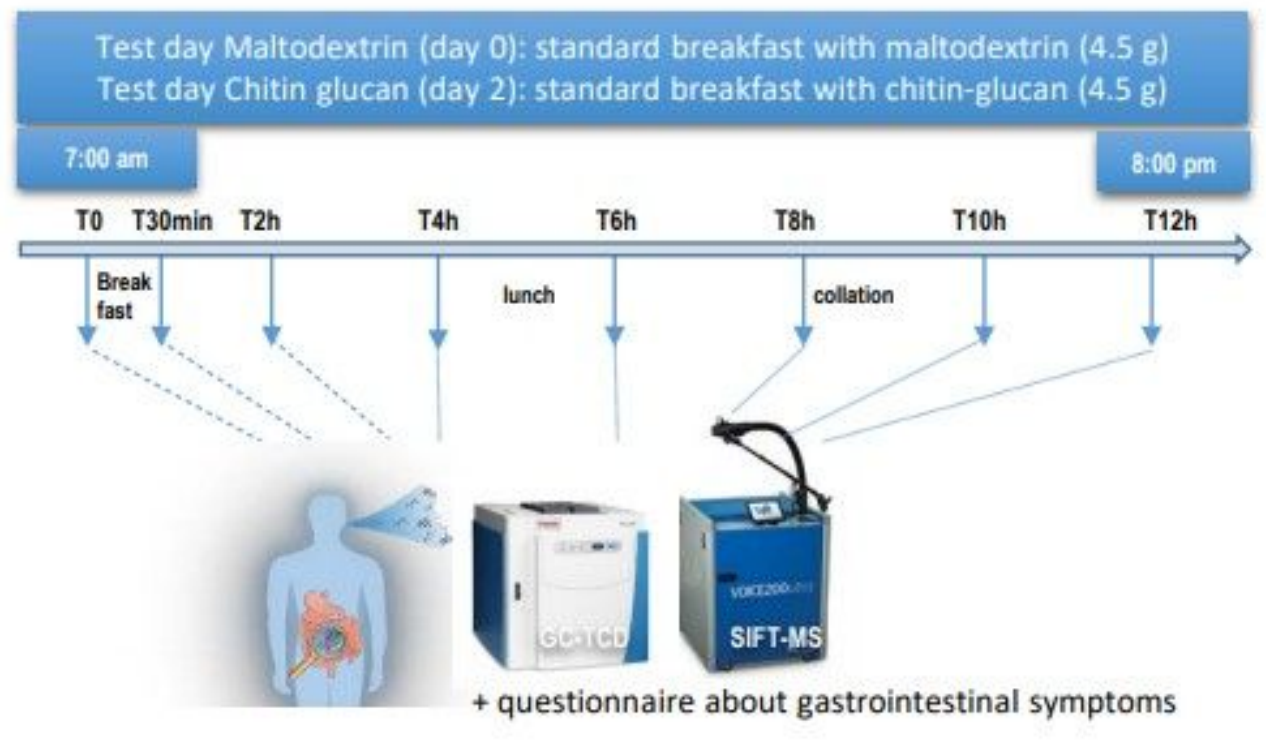

\section{Figure 1}

Overview of the study design. 

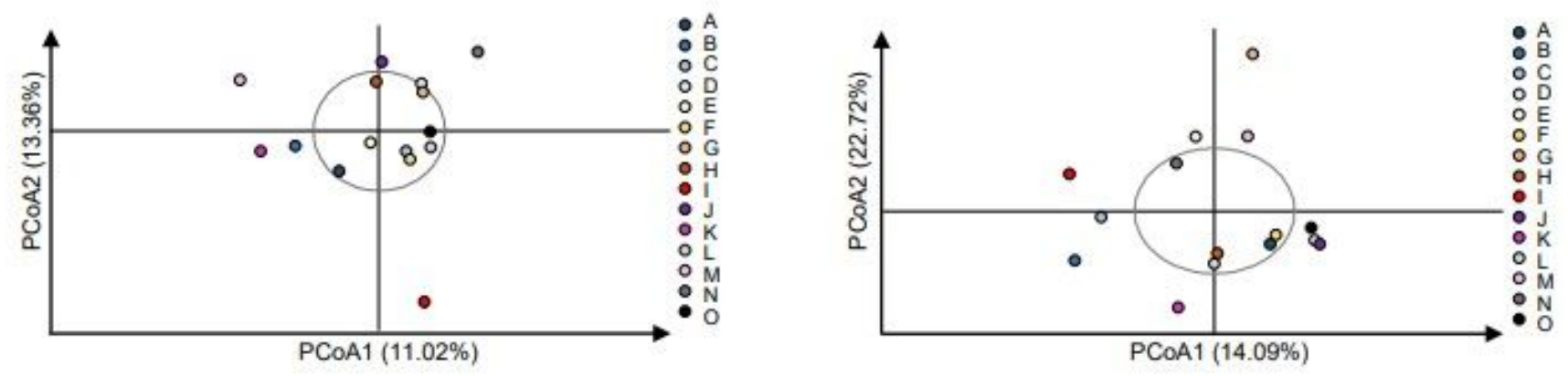

B
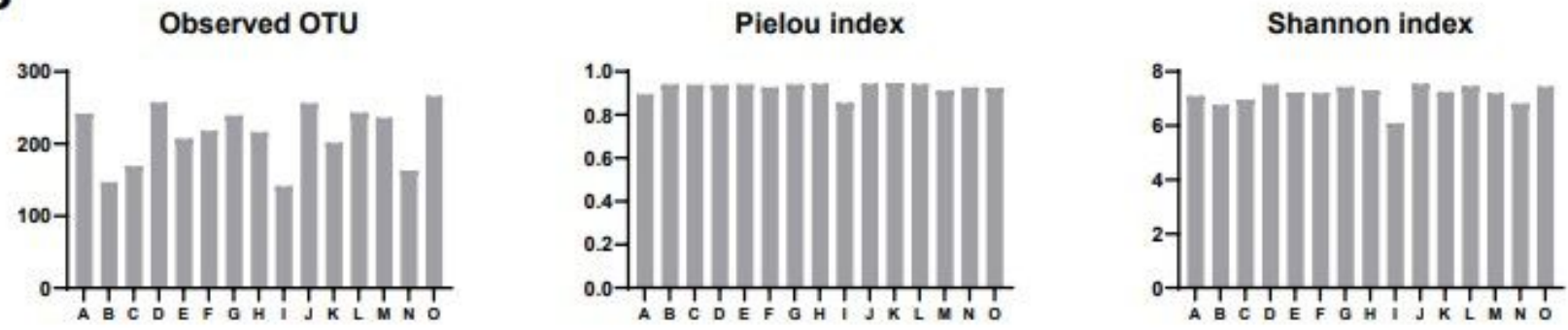

C

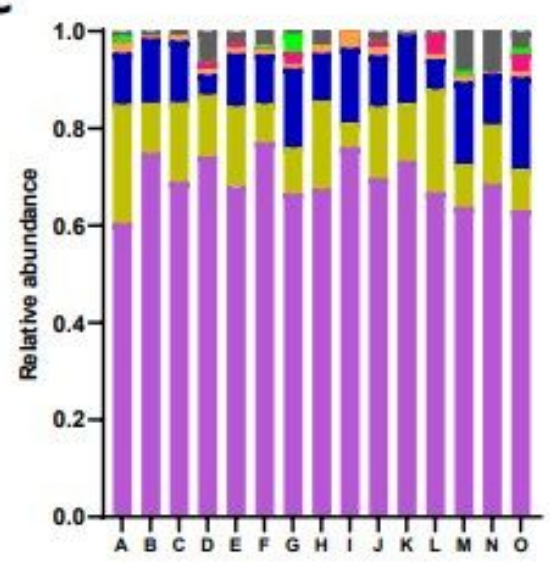

D
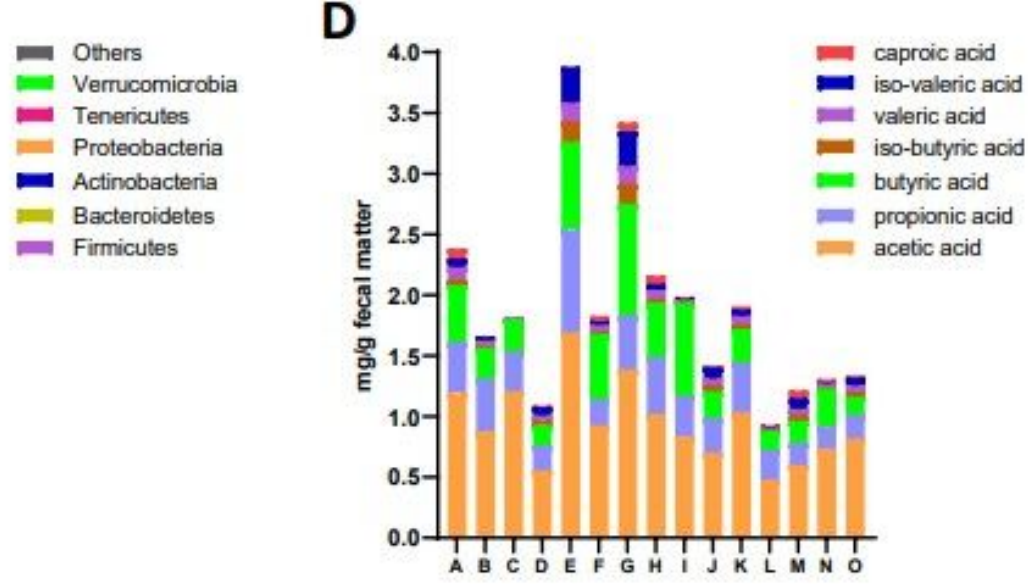

\section{Figure 2}

Gut microbiota and short-chain fatty acids (SCFA) composition in faecal matter of 15 healthy subjects

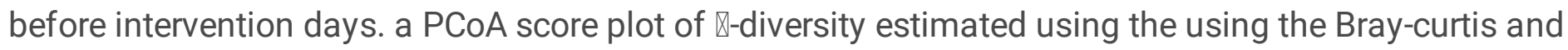
unweighted uniFrac indexes. $\mathrm{b} \mathbb{\Delta}$-diversity indexes related to bacterial richness (Observed OTU), evenness (Pielou) or both (Shannon) for each subject. c Bar plots of relative abundance of phylum levels accounting for more than $0.5 \%$ for each subject. $d$ Bar plots of SCFA concentration for each subject. 

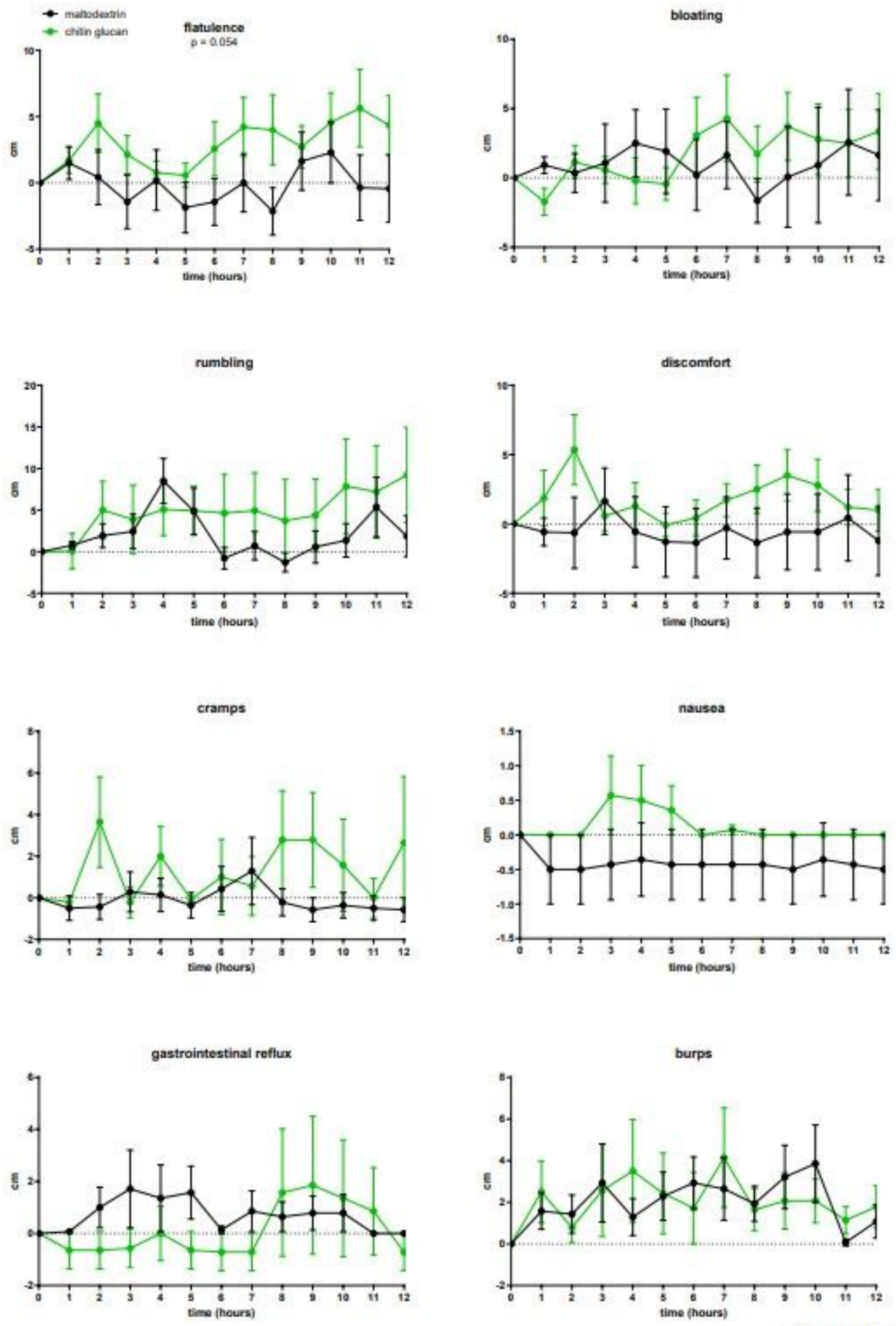

FIGURE 3

\section{Figure 3}

Gastrointestinal tolerance assessed by visual analog scale of 15 healthy subjects about 8 symptoms after chitin-glucan intake of maltodextrin intake. Data are means \pm SEM $(p>0.05$; matched-pairs Wilcoxon signed-rank test on net AUC). 
A PCA of the MS-fragments before interventions

(day 0 - time $0 \mathrm{~h}$ - fasting state)

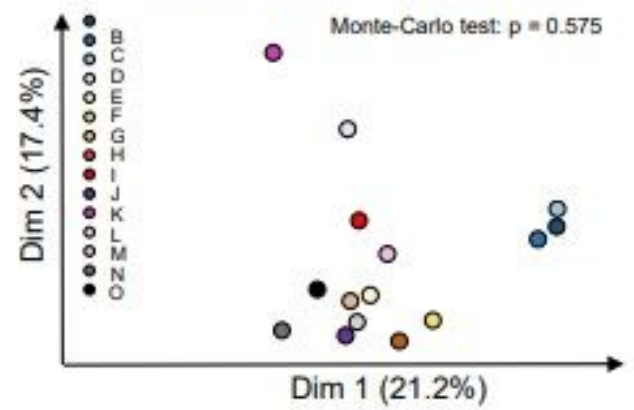

B

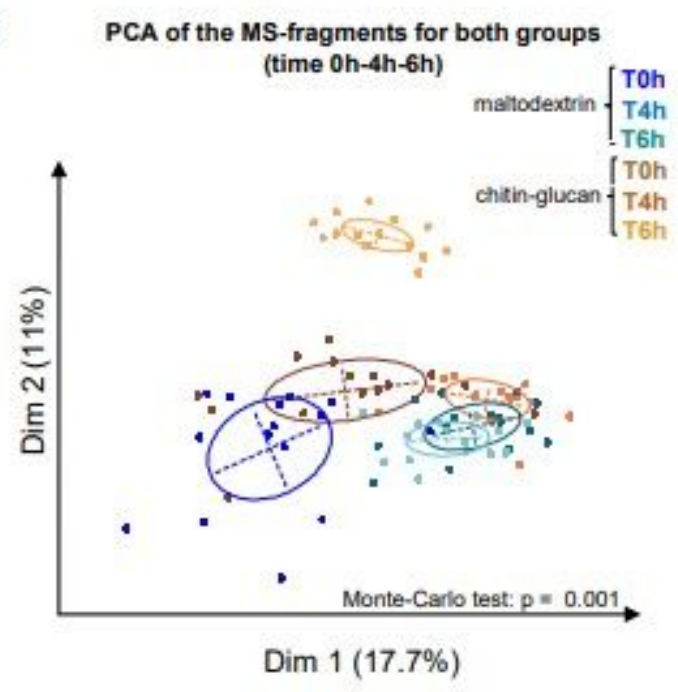

C

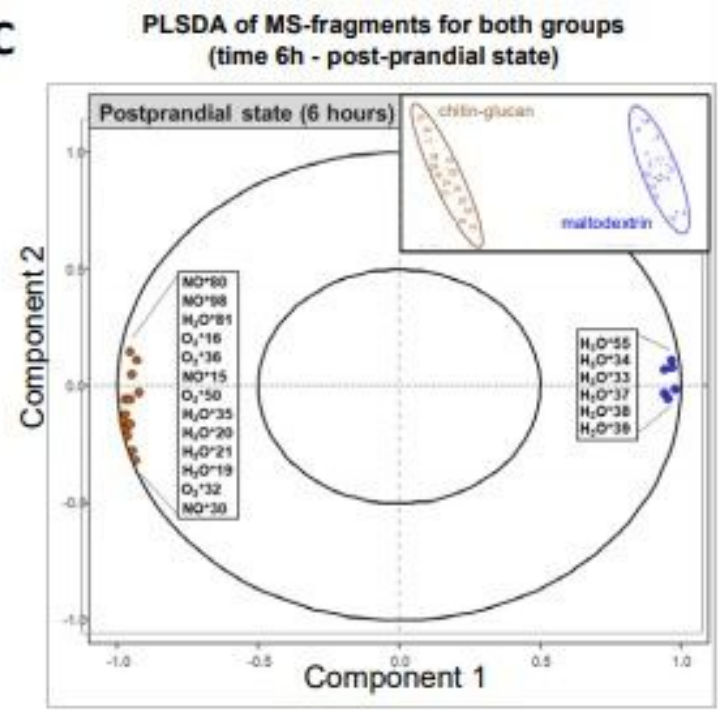

\section{Figure 4}

Untargeted analysis of breath from 15 healthy subjects after chitin-glucan and maltodextrin intake. PCA score plot of the MS-fragments from the SIFT-MS spectra (from $\mathrm{H} 3 \mathrm{O}+, \mathrm{NO}+$ and $\mathrm{O} 2+$ ionizations) obtained in fasted state (time $0 \mathrm{~h}$ ) the first test day (a) or in fasted state (time 0h), in pre-prandial state (time 4h) and in post-prandial state (time 6h) (b). PLSDA (with cutoff 0.9) of 408 MS-fragments after chitin-glucan intake and maltodextrin intake at time $6 \mathrm{~h}$ (c). Statistical analysis was assessed by a Monte Carlo rank test. 

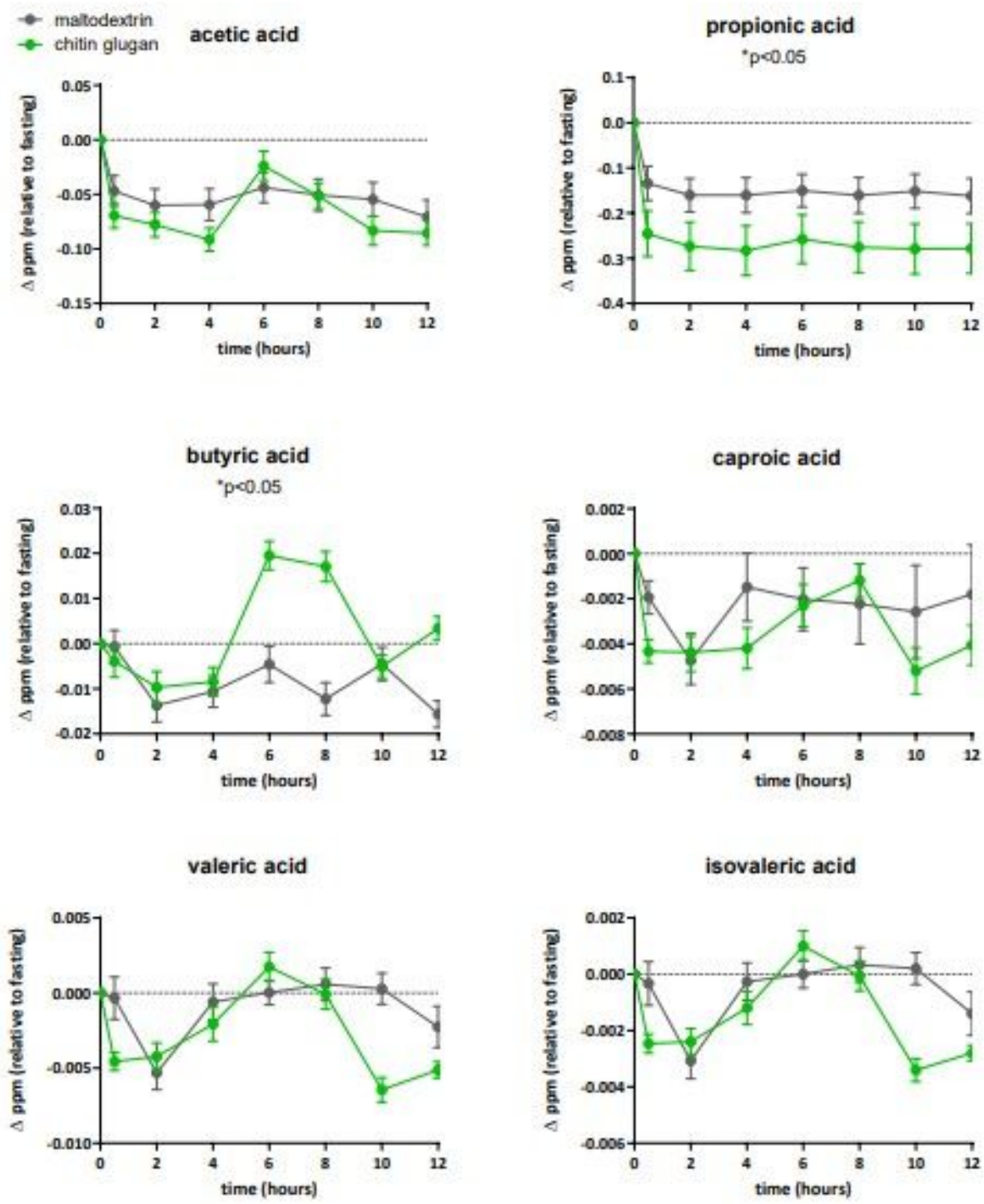

\section{Figure 5}

Targeted SCFA concentrations (changes from baseline) exhaled in breath of healthy subjects in response to chitin-glucan and maltodextrin intake. Data are means \pm SEM $\left({ }^{*} \mathrm{p}<0.05\right.$; matched-pairs Wilcoxon signed-rank test on net AUC). 

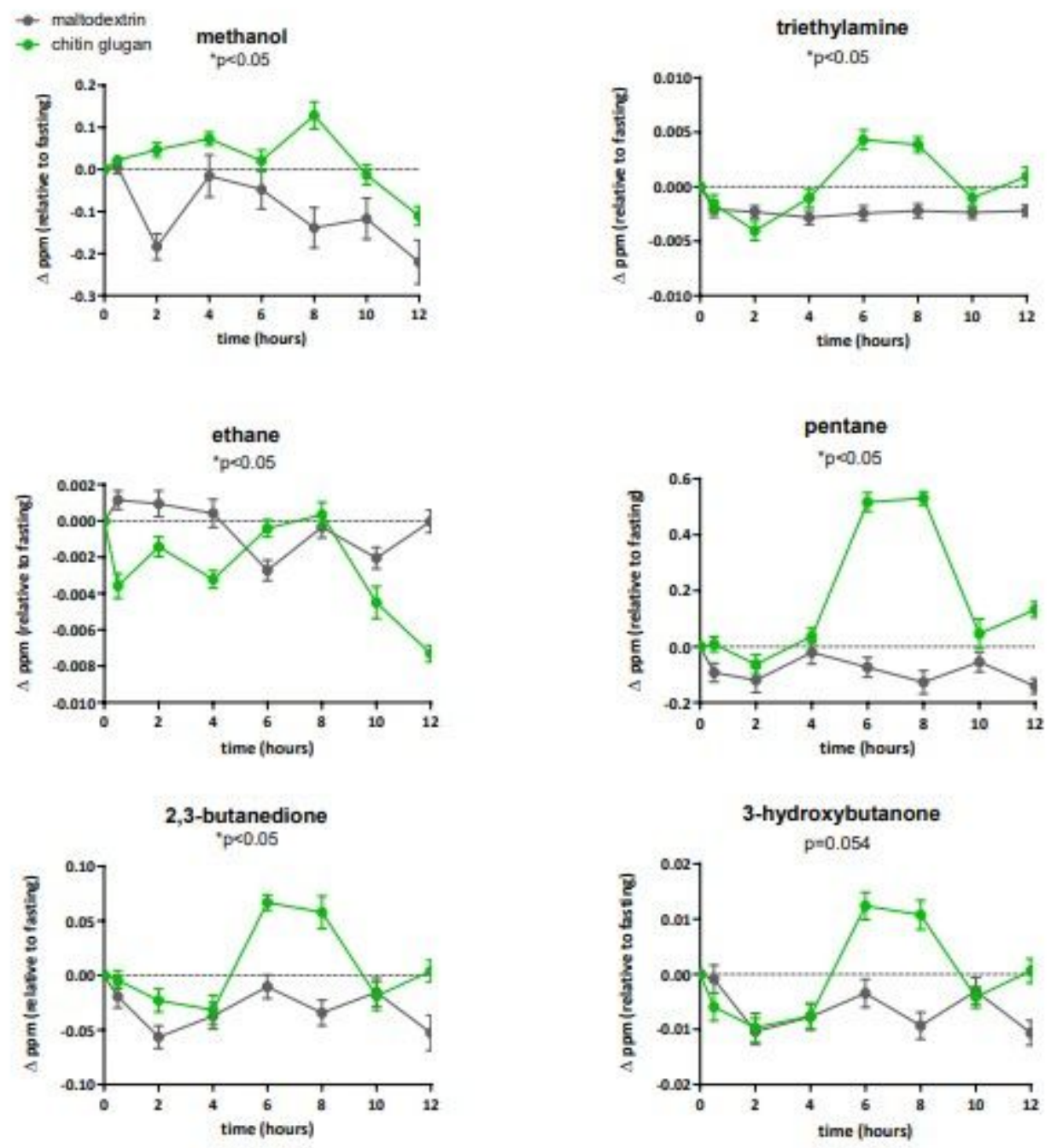

\section{Figure 6}

Targeted BVM concentrations (changes from baseline) exhaled in breath of healthy subjects significantly changed after chitin-glucan intake compared to maltodextrin intake. Data are means \pm SEM $\left({ }^{*} \mathrm{p}<0.05\right.$; matched-pairs Wilcoxon signed-rank test on net area under the curve).

\section{Supplementary Files}

This is a list of supplementary files associated with this preprint. Click to download.

- NeyrinckRodriguezdataset1.xlsx

- NeyrinckRodriguezSupplementaryfile.docx 